Abstract EPV122/\#421 Table 1 Multivariable analysis for causespecific survival after isolated lymphatic recurrence: factors independently associated with cause-specific survival

\begin{tabular}{|c|c|c|c|}
\hline Characteristic & No. of events per level & $\begin{array}{c}\text { Adjusted } \\
\text { HR (95\% Cl) }\end{array}$ & $\mathbf{P}$ \\
\hline FIGO grade & & & 0.007 \\
\hline 1 & $5 / 14$ & Reference & \\
\hline 2 & $12 / 16$ & $5.11(1.68 .15 .52)$ & \\
\hline 3 & 33,40 & $5.10(1.79,14.51)$ & \\
\hline Pelvic and paraaortic ILR & & & 0.002 \\
\hline No & 38156 & Reference & \\
\hline Yes & $12 / 14$ & $3.08(1.52,6.21)$ & \\
\hline Concomitant vaginal recurrence & & & $<0.001$ \\
\hline No & 4666 & Refurence & \\
\hline Yes & 44 & $8.21(2.50,26.97)$ & \\
\hline Treatment of ILR & & & 0.03 \\
\hline Observation or hormonal therapy only & $13 / 17$ & $2.60(1.09,6.19)$ & \\
\hline Chemotherapy and or radiotherapy & $28 / 34$ & $2.75(1.27,5.94)$ & \\
\hline Surgery \pm other treatments & 9/19 & Reference & \\
\hline
\end{tabular}

predictors of poor CSS after ILR. The choice to surgically treat ILR in some patients was associated with improved CSS.

\section{EPV123/\#437 RISK OF LEIOMYOSARCOMA IN PATIENTS UNDERGOING HYSTERECTOMY FOR PRESUMED BENIGN DISEASE}

M Longo*, E Ervas, A Lembo, V Artuso, A Baranzini, E Galati, F Ghezzi, J Casarin. Women's and children hospital F. Del Ponte Hospital - University of Insubria, Obstetrics and Gynecology, Varese, Italy

\subsection{6/ijgc-2021-IGCS.193}

Objectives To estimate the incidence and to identify risk factors of leiomyosarcoma among women undergoing hysterectomy for presumed benign disease.

Methods This is a retrospective single-center study of consecutive patients who underwent total hysterectomy with benign indications at Del Ponte Hospital (Varese) between 01/01/2000 and $31 / 12 / 2019$. Data were manually collected by operative records and institutional surgical reports, including demographic and histopathologic characteristics. Factors associated with the occurrence of unexpected uterine leiomyosarcoma (uLMS) were searched. Stratification by age, menopausal status and uterine weight was performed.

Results Overall, 4428 patients were included in the analysis and $24(0,54 \%)$ had a final diagnosis of uLMS. Among 2936 patients with preoperative indication of uterine fibroids, the rate of uLMS was $0,99 \%$. The increase of age at surgery resulted to be positively associated with the incidence of uLMS (from $0.09 \%$ in patients $<45$ yo to $1.97 \%$ in patients $>75$ yo; $p=0.01)$. The absolute risk of LMS increased in postvs. premenopausal patients $(1.27 \%$ vs. $0.25 \% ; \mathrm{p}=0.001)$. Increase in uterine weight was also associated with higher risk of uLMS $(\mathrm{p}<0.001)$.

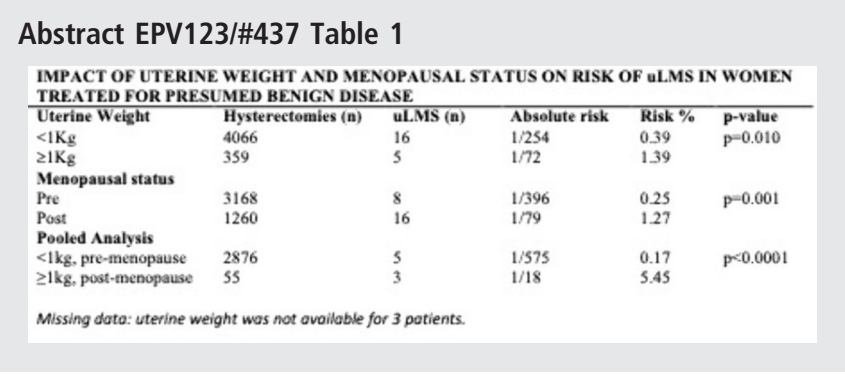

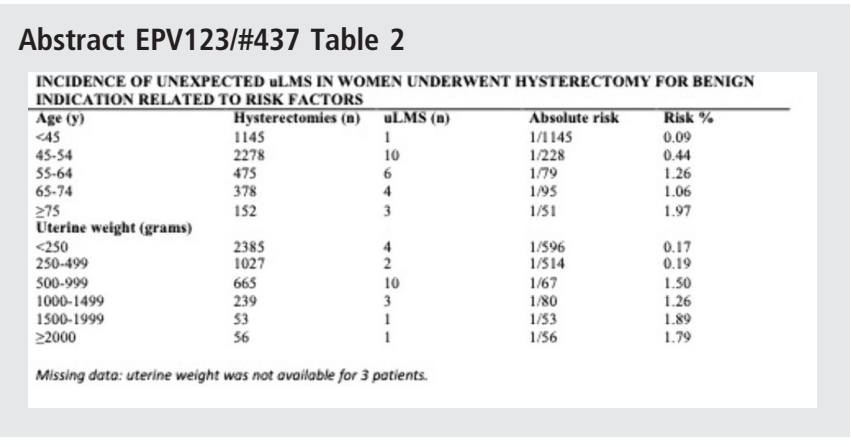

The pooled analysis included menopausal status (pre vs. post) and uterine weight ( $<1 \mathrm{~kg}$ vs. $>1 \mathrm{~kg}$ ); post-menopausal women with uterus weighting $1 \mathrm{~kg}$ or more had an absolute risk of uLMS of $5.45 \%$.

Conclusions The overall risk of uLMS in women undergoing hysterectomy for presumed benign indication is low. However, there is a significant increased risk in post-menopausal patients with enlarged uteri.

\section{EPV124/\#439 NEOADJUVANT CHEMOTHERAPY FOLLOWED BY SURGERY FOR ADVANCED-STAGE ENDOMETRIAL CANCER}

${ }^{1} \mathrm{H}$ Khaldi; ${ }^{*},{ }^{2} \mathrm{MT}$ Alsayed, ${ }^{3} \mathrm{~A}$ Al -Ansari, ${ }^{2} \mathrm{M}$ Abu-Jubara, ${ }^{4} \mathrm{H}$ Almalik, ${ }^{5} \mathrm{~S}$ Alhyassat, ${ }^{1} \mathrm{JJO}$ Herod. 'Hamad Medical Corporation, Women Wellness and Research Center, Doha, Qatar, ${ }^{2}$ Hamad Medical Corporation, Women Wellness and Research Center, DOHA, Qatar; ${ }^{3}$ WWRC, Obs and Gyn, Doha, Qatar; ${ }^{4}$ NCCCR, Oncology, Doha, Qatar; ${ }^{5}$ Hamad Medical Coorporation, Pathology, Doha, Qatar

\subsection{6/ijgc-2021-IGCS. 194}

Objectives Neoadjuvant chemotherapy (NACT) plus interval debulking surgery (IDS) is a treatment strategy for ovarian cancer patients with unresectable disease or poor performance status. It has also used for the treatment of advanced endometrial cancer (ECa) and a survival benefit has been shown. This study reviews our single-institution experience with NACT and surgery for advanced endometrial cancer.

Methods Data were collected retrospectively about patients with ECa treated January 2015-March 2021. Outcome measures include response; survival; and treatment-related morbidity.

Results There were 18 patients aged 39-72yrs. Data is complete for 16 (two had surgery overseas). Histological type was: endometrioid (72\%); serous (22\%); mixed (6\%). 33\% were stage IV; $45 \%$ stage III; 22\% stage II. All patients received Carboplatin/Paclitaxel chemotherapy. Two also received radiotherapy before surgery. Patients received between 2-6 cycles of chemotherapy. Fifteen patients $(83.3 \%)$ had optimal debulking surgery and one sub-optimal debulking. One patient was lost to follow-up. Another expired before surgery due to septic shock. Data regarding survival is available for 14/18 patients. One has died. Thirteen patients are alive with survival of $6-48 \mathrm{mth}$. Two patients are alive with recurrence. Eleven are alive without recurrence. Overall median survival is currently $20 \mathrm{mth}$. $83 \%$ had no significant complications; $11 \%$ had wound infection; one patient died from septic shock.

Conclusions NACT and IDS delivers high rates of optimal debulking in patients with advanced stage ECa. There are acceptable levels of morbidity. This study suggests that NACT 
followed by IDS is at least a non-inferior strategy for patients with advanced ECa who are unsuitable for primary surgery.

\section{EPV125/\#443 THE PREVALENCE OF UTERUS CANCER IN UZBEKISTAN}

S Djanklich*, Z Sabirdjanova. Republican Specialized Scientific-Practical Medical Center of Oncology and Radiology, Gynecological, Tashkent, Uzbekistan

\subsection{6/ijgc-2021-IGCS. 195}

Objectives To estimate the prevalence of endometrial cancer in Uzbekistan over the last 3 years.

Methods We collected uterine cancer statistical indicators from official statistics in Uzbekistan for the years 2018-2020.

Results Analyzed data of uterina cancer (UC) in 2018-20 years showed that $641(1.4), 640(1,9)$ and $609(1,8)$ UC patients were identified in the Republic, respectively. In 2018 year 315 patients were from the country - side. The patients were into stages as follows: I stage - $31.2 \%$, II stage - 39.9\%, III stage $16.1 \%$, IV - 5.3\%. The mortality rate in 2018 was 0.7 (234 patients) and the 5 -year survival rate consisted $47.3 \%$. The patients were into stages as follows: I stage - 34.8\%, II stage - 41.7\%, III stage - $14.4 \%$, IV stage - 3.1\%. The mortality rate in 2020 was 0.8 (256 patients) and the 5-year survival rate consisted $49.5 \%$. In 2020 the patients were into stages as follows: I stage $-35,8 \%$, II stage $-41,7 \%$, III stage $-11,3 \%$, IV stage - 4,4\%. 568 patients were from the country-side. 5year survival rate consisted $48,7 \%$. The mortality rate in 2020 was 0,8 (274).

Conclusions The morbidity of UC in Uzbekistan has not tend to decrease and requires primary care physicians to promote a healthy lifestyle, a more careful approach to all types of uterine bleeding at women of both fertile and menopausal age. Timely putting diagnosis and treatment of endometrial hyperplastic processes will significantly reduce the number of women at risk for UC.

\section{EPV126/\#448 UNDIFFERENTIATED AND DEDIFFERENTIATED CARCINOMA OF THE ENDOMETRIUM: CLINICOPATHOLOGIC FEATURES AND IMPLICATIONS FOR PROGNOSTICATION AND MANAGEMENT}

${ }^{1} \mathrm{SJ}$ Mah*, ${ }^{2} \mathrm{JY}$ Dong, ${ }^{1} \mathrm{JMV}$ Nguyen, ${ }^{1} \mathrm{~L}$ Bernard, ${ }^{3} \mathrm{~A}$ Lytwyn, ${ }^{3} \mathrm{~J}$ Maxwell, ${ }^{3} \mathrm{D}$ Daya, ${ }^{3} \mathrm{M}$ Sur, ${ }^{1} \mathrm{~L}$ Eiriksson, ${ }^{1} \mathrm{C}$ Reade, ${ }^{1} \mathrm{~V}$ Carlson, ${ }^{1} \mathrm{~L}$ Elit, ${ }^{1} \mathrm{~W}$ Jimenez. ${ }^{1} \mathrm{McM}$ aster University, Juravinski Hospital and Cancer Centre, Gynecologic Oncology, Hamilton, Canada; ${ }^{2}$ Michael G. DeGroote School of Medicine, McMaster University, Medicine, Hamilton, Canada; ${ }^{3}$ McMaster University, Pathology and Molecular Medicine, Hamilton, Canada

\subsection{6/ijgc-2021-IGCS.196}

Objectives Undifferentiated and dedifferentiated endometrial carcinomas (UEC/DDEC) are rare, high grade, and have only been increasingly recognized within the past decade. Studies of their behavior and response to adjuvant to guide prognostication and management are limited. We present the management experience of a single institution.

Methods Using the Juravinski Hospital electronic medical record, we identified all patients with UEC or DDEC treated at our institution from January 2005-December 2020. Clinical information was obtained by chart review.

Results We identified 35 patients with UEC/DDEC; 15 UEC, 20 DDEC. Mean age was 66 years. Only $25.1 \%$ had preoperative endometrial biopsy concordant with final pathology despite $87.5 \%$ review by gynecologic pathologists. Stage distribution was $37.1 \%$ stage I, $14.3 \%$ stage II, $14.3 \%$ stage III, $34.3 \%$ stage IV. $7 / 33$ (21.2\%) had gross residual after surgery; 4 received adjuvant carboplatin-paclitaxel chemotherapy with 2 progressions, 1 partial response and 1 complete response (ORR 50\%). Mean PFS was 11.7 \pm 9.3 months. Fifteen patients had progressive or recurrent disease-of these, 4 were treated with radiation, 3 with chemotherapy (adriamycin, carboplatin-paclitaxel, doxorubicin), and all 7 progressed on treatment. The most common site of recurrence was widely disseminated disease (54.5\%), followed by nodal (18.2\%) and chest (18.2\%). Mean OS was as follows by stage: stage I-II completely resected, 43 months; stage III completely resected, 19 months; stage IV, suboptimally debulked or inoperable, 20 months.

Conclusions UEC/DDEC are aggressive tumours with poor prognoses and remain challenging to diagnose on preoperative biopsy. Platinum-based adjuvant chemotherapy may have some efficacy, however, recurrences respond poorly to salvage.

\section{EPV127/\#500 IMPACT OF COMPUTED TOMOGRAPHY- DETERMINED SARCOPENIA AND ARTIFICIAL INTELLIGENCE-DRIVEN WAIST SKELETAL MUSCLE VOLUME ON SURVIVAL OUTCOME IN ENDOMETRIAL CANCER}

${ }^{1} \mathrm{~A} \mathrm{Seol}^{*},{ }^{1} \mathrm{SI} \mathrm{Kim},{ }^{2} \mathrm{HS}$ Kim, ${ }^{1} \mathrm{HH}$ Chung, ${ }^{3} \mathrm{~J}-\mathrm{W}$ Kim, ${ }^{1} \mathrm{M}$ Lee. ${ }^{1}$ Seoul National University College of Medicine, Department of Obstetrics and Gynecology, Seoul, Korea, Republic of; ${ }^{2}$ Seoul National University Hospital, Department of Obstetrics and Gynecology, Seoul, Korea, Republic of; ${ }^{3}$ Seoul National University College of Medicine, Obstetrics and Gynecology, Seoul, Korea, Republic of

\subsection{6/ijgc-2021-IGCS.197}

Objectives To investigate the impact of computed tomography (CT)-determined sarcopenia and body composition on survival outcomes in patients with endometrial cancer.

Methods We retrospectively identified patients diagnosed with endometrial cancer between 2014 and 2018. Using an artificial intelligence-based tool, the skeletal muscle area $\left(\mathrm{cm}^{2}\right)$ at the third lumbar vertebra (L3) and the skeletal muscle volume $\left(\mathrm{cm}^{3}\right)$ at the waist level from pre-treatment CT scans were measured. These values were converted to the L3 skeletal muscle index (SMI) index and volumetric SMI by normalisation. The relationships between L3, volumetric SMIs, and survival outcomes were evaluated.

Results Altogether, data of 385 patients were analysed. The mean patient age was 55.5 years. Applying the well-known cut-off value for sarcopenia to the L3 SMI, sarcopenia $(<39.0$ $\left.\mathrm{cm}^{2} / \mathrm{m}^{2}, \mathrm{n}=177\right)$ and non-sarcopenia $\left(\geq 39.0 \mathrm{~cm}^{2} / \mathrm{m}^{2}, \mathrm{n}=208\right)$ groups showed similar progression-free survival (PFS; $\mathrm{P}=0.335)$ and overall survival (OS; $\mathrm{P}=0.241)$. Using the median value, the low-volumetric SMI group $\left(<206.0 \mathrm{~cm}^{3} / \mathrm{m}^{3}\right.$, $\mathrm{n}=192$ ) showed significantly worse PFS (3-year survival rate, $77.3 \%$ vs. $88.8 \% ; \mathrm{P}=0.004)$ and $\mathrm{OS}$ (3-year survival rate, $92.8 \%$ vs. $99.4 \% ; \mathrm{P}=0.003)$ than the high-volumetric SMI group $\left(\geq 206.0 \mathrm{~cm}^{3} / \mathrm{m}^{3}, \mathrm{n}=193\right)$. In multivariate analyses adjusted for baseline body mass index and other factors, lowvolumetric SMI was identified as an independent poor prognostic factor for PFS (adjusted HR, 1.762; 95\% CI, 1.0512.953; $\mathrm{P}=0.032$ ) and $\mathrm{OS}$ (adjusted HR, 5.964; 95\% CI, 1.296-27.448; $\mathrm{P}=0.022$ ). 